\title{
Optical properties of (AlxGa1-x)(0.52)In0.48P at the crossover from a direct-gap to an indirect-gap semiconductor
}

Dörr, U.; Schwarz, W.; Wörner, A.; Westphäling, R.; Dinger, A.; Kalt, H.; Mowbray, D.J.; Hopkinson, M.; Langbein, Wolfgang Werner

Published in:

Journal of Applied Physics

Link to article, DOI:

$10.1063 / 1.366963$

Publication date:

1998

Document Version

Publisher's PDF, also known as Version of record

Link back to DTU Orbit

Citation (APA):

Dörr, U., Schwarz, W., Wörner, A., Westphäling, R., Dinger, A., Kalt, H., Mowbray, D. J., Hopkinson, M., \& Langbein, W. W. (1998). Optical properties of (AlxGa1-x)(0.52)In0.48P at the crossover from a direct-gap to an indirect-gap semiconductor. Journal of Applied Physics, 83(4), 2241-2249. https://doi.org/10.1063/1.366963

\section{General rights}

Copyright and moral rights for the publications made accessible in the public portal are retained by the authors and/or other copyright owners and it is a condition of accessing publications that users recognise and abide by the legal requirements associated with these rights.

- Users may download and print one copy of any publication from the public portal for the purpose of private study or research.

- You may not further distribute the material or use it for any profit-making activity or commercial gain

- You may freely distribute the URL identifying the publication in the public portal 


\title{
Optical properties of $\left(\mathrm{Al}_{x} \mathrm{Ga}_{1-x}\right)_{0.52} \mathrm{In}_{0.48} \mathrm{P}$ at the crossover from a direct-gap to an indirect-gap semiconductor
}

\author{
U. Dörr, ${ }^{\text {a) }}$ W. Schwarz, A. Wörner, R. Westphäling, A. Dinger, and H. Kalt \\ Institut für Angewandte Physik, Universität Karlsruhe, D-76128 Karlsruhe, Germany \\ D. J. Mowbray \\ Department of Physics, University of Sheffield, Sheffield S3 7RH, United Kingdom \\ M. Hopkinson \\ Department of Electronic and Electrical Engineering, EPSRC Central Facility for III-V Materials, \\ University of Sheffield, Sheffield S1 3JD, United Kingdom \\ W. Langbein \\ Mikroelektronik Centret, The Technical University of Denmark, Building 345 east, DK-2800 Lyngby, \\ Denmark
}

(Received 31 July 1997; accepted for publication 26 October 1997)

\begin{abstract}
The optical properties and the dynamics of excitons and the electron-hole plasma have been studied in disordered $\left(\mathrm{Al}_{x} \mathrm{Ga}_{1-x}\right)_{0.52} \mathrm{In}_{0.48} \mathrm{P}$ near to the direct-to-indirect band gap crossover. In particular we have investigated three epitaxial layers grown by solid-source molecular beam epitaxy with varying Al content $x$. Two of them have compositions in the immediate vicinity of the crossover point, the other is assigned to the indirect-gap regime. Both direct and indirect recombination processes contribute to the photon emission from the material. Since the relative importance of the different recombination processes depends strongly on temperature, excitation intensity, and excitation pulse duration, the processes can be identified by changing these parameters. As a result, we can determine the relative alignment of the conduction band minima and the distribution of the electrons among them. At high excitation levels the two crossover samples show stimulated emission at a photon energy of $\sim 2.29 \mathrm{eV}$, i.e., in the green spectral range. Using the variable stripe length method, we find an optical gain of up to $\sim 600 \mathrm{~cm}^{-1}$ at excitation levels of $\sim 350 \mathrm{~kW} / \mathrm{cm}^{2}$. Stimulated emission involves direct recombination. This conclusion is reached from the experiments and from line-shape modeling, including a self-consistent treatment of populations and renormalization of the conduction band minima. (C) 1998 American Institute of Physics.
\end{abstract} [S0021-8979(98)07403-9]

\section{INTRODUCTION}

The quaternary compound $\left(\mathrm{Al}_{x} \mathrm{Ga}_{1-x}\right)_{0.52} \mathrm{In}_{0.48} \mathrm{P}$ is of considerable technological interest as it has the largest direct band gap of any III-V semiconductor lattice matched to GaAs. Laser emission in the red spectral region from $\left(\mathrm{Al}_{x} \mathrm{Ga}_{1-x}\right)_{0.52} \mathrm{In}_{0.48} \mathrm{P}$ laser structures has been demonstrated. ${ }^{1}$ Highly efficient light emitting diodes (LEDs) emitting in the green spectral range have been produced ${ }^{2}$ and are now available commercially. Furthermore, $\left(\mathrm{Al}_{x} \mathrm{Ga}_{1-x}\right)_{0.52} \mathrm{In}_{0.48} \mathrm{P}$ is used as the barrier material in practically all laser structures with ternary $\mathrm{Ga}_{0.52} \mathrm{In}_{0.48} \mathrm{P}$ active layers.

Despite the numerous applications, the optical properties of this quaternary alloy especially for high Al content $x$, are still not well known. The direct band gap increases with increasing $x$ while the indirect gap involving the $X$-point minima hardly varies with composition. Therefore the fundamental gap changes its character from direct to indirect at a crossover composition of $x_{e} \sim 0.5 .^{3}$ Mowbray et al. determined this transition composition using photoluminescence

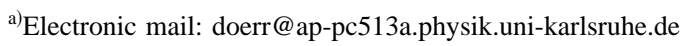

(PL) and photoluminescence excitation (PLE) spectroscopy. ${ }^{3}$ Unfortunately the crossover appears to be a limiting factor for the emission energy of photonic devices. Nelson et al. ${ }^{4}$ found that the luminescence intensity of $\left(\mathrm{Al}_{x} \mathrm{Ga}_{1-x}\right)_{0.52} \mathrm{In}_{0.48} \mathrm{P}$ decreases dramatically when the $\mathrm{Al}$ concentration approaches the crossover value. From theoretical considerations, consistent with pressure-dependent PL measurements, they concluded that this decrease is due to nonradiative processes occurring at the indirect gap. As the pressure-induced direct-indirect band gap crossover produces a similar decrease of radiative efficiency for $\mathrm{Al} \mathrm{com-}$ positions considerably below the crossover value, the nonradiative recombination center is not necessarily related to an increasing $\mathrm{Al}$ composition. All the samples that they investigated showed only direct $(\Gamma-\Gamma)$ recombination. No PL was observed beyond crossover.

In addition to such composition dependent properties, the CuPt-type ordering which occurs in metalorganic vapor phase epitaxy (MOVPE) grown $\left(\mathrm{Al}_{x} \mathrm{Ga}_{1-x}\right)_{0.52} \mathrm{In}_{0.48} \mathrm{P}$ prepared under certain growth conditions has strong effects on the band structure. ${ }^{5,6}$ Yamashita et $_{\text {al. }}{ }^{7}$ have reported an order-induced indirect-to-direct band-gap transition. 
In the present work we focus our attention on the optical properties of disordered $\left(\mathrm{Al}_{x} \mathrm{Ga}_{1-x}\right)_{0.52} \mathrm{In}_{0.48} \mathrm{P}$ near the crossover composition. The sample composition relative to the crossover point is determined by low-intensity $\mathrm{cw}$ PL and PLE (Sec. III A). We identify the optical transitions as being of direct or direct nature and investigate their relative importance as a function of lattice temperature (Sec. III B). The optical properties under high excitation conditions are discussed in Sec. IV. We treat the dense carrier system in terms of an electron-hole plasma where the electrons are distributed between the $\Gamma$ and $X$ minima. Many-particle effects, for example, band-gap renormalization and stimulated emission, are included in our treatment (Sec. IV A). As a first experimental step, we study the contributions of direct and indirect recombination with increasing carrier density (Sec. IV B) and their temporal evolutions (Sec. IV C). The origin of the stimulated emission and optical gain is discussed comprehensively by comparison to quasistationary and timeresolved high-excitation PL (Sec. IV D).

\section{SAMPLES AND EXPERIMENTAL PROCEDURES}

Three $\left(\mathrm{Al}_{x} \mathrm{Ga}_{1-x}\right)_{0.52} \mathrm{In}_{0.48} \mathrm{P}$ samples consisting of epitaxial layers of 1.3-1.5 $\mu \mathrm{m}$ thickness have been investigated. The InP mole fraction of 0.48 ensures lattice match to GaAs while the $\mathrm{Al}: \mathrm{Ga}$ ratio has no significant effect on the lattice constant of the quaternary compound. The samples were grown by solid-source molecular-beam epitaxy on undoped (001) GaAs substrates. Under these growth conditions no CuPt-type ordering occurs. Group-III compositions were determined from reflection high-energy electron diffraction (RHEED) oscillation measurements performed during the growth of the binary compounds on appropriate substrates. Samples 1, 2, and 3 have nominal Al concentrations of 0.48 , 0.52 , and 0.58 , respectively.

Optical characterization was carried out by PL and PLE. PL was excited with light from a $200 \mathrm{~W} \mathrm{Hg}$ lamp, dispersed by a prism monochromator, and detected by an optical multichannel analyzer (OMA). In the case of temperature dependent PL measurements the $514.5 \mathrm{~nm}$ line of an $\mathrm{Ar}^{+}$laser was used as an excitation source. PLE was excited with light from a $150 \mathrm{~W}$ Xe lamp which was dispersed by a grating monochromator. The signal was detected by a photomultiplier linked to a lock-in amplifier. Raman measurements were performed in backscattering geometry using the 488 $\mathrm{nm}$ line of an $\mathrm{Ar}^{+}$laser.

Excitation intensity dependent luminescence measurements were carried out with short-pulsed excitation (pulse duration $100 \mathrm{fs}$ ) but time integrated detection. For the study of high excitation effects, two types of excitation conditions were used: (a) quasistationary excitation obtained with the ns pulses of a nitrogen pumped dye laser, for the high excitation PL and the variable stripe length method measurements and (b) nonstationary excitation with the pulses of an excimer pumped quenched transient dye laser system (pulse duration $100 \mathrm{ps}$, repetition rate $10 \mathrm{~Hz}$ ) for the time resolved photoluminescence measurements. Time resolved PL was detected by a single shot streak camera. For the high excitation PL and variable stripe length measurements, an OMA detector

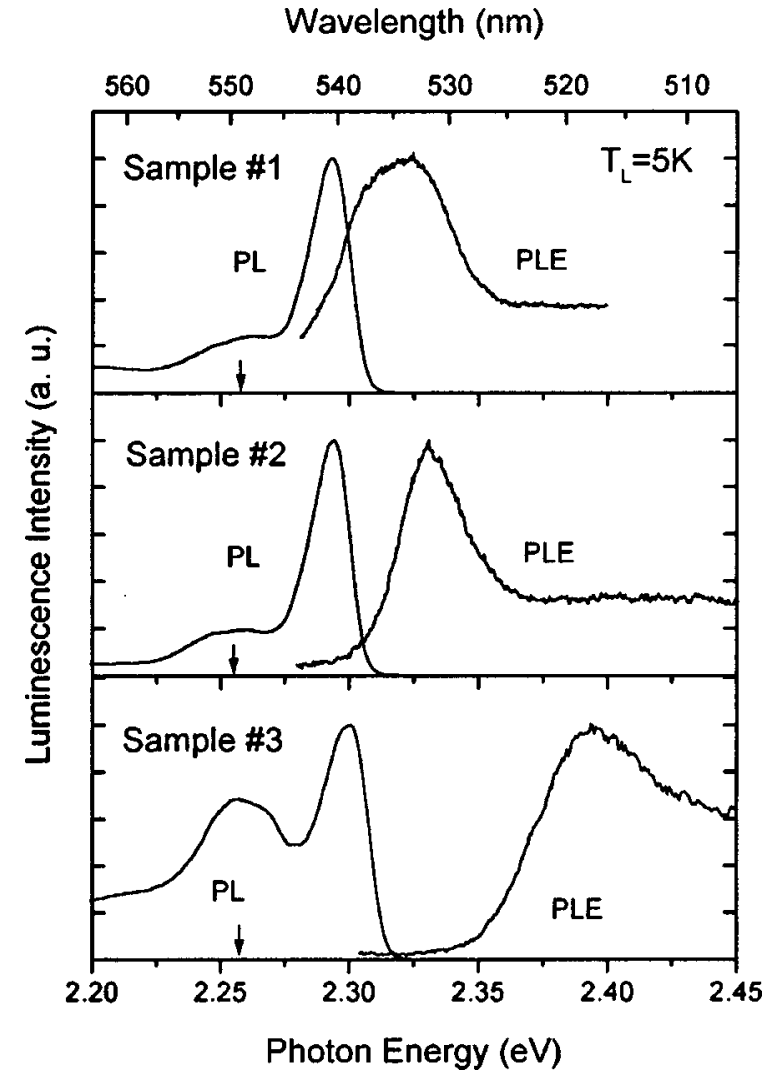

FIG. 1. PL and PLE spectra of all the samples recorded at $T=5 \mathrm{~K}$. In order to avoid detection of scattered excitation light, the PLE spectra were recorded at the low-energy part of the PL (the detection energies are indicated by arrows).

was used. All experiments, except the Raman $(80 \mathrm{~K})$ and the temperature-dependent PL measurements, were carried out at low temperatures of $5 \mathrm{~K}$.

\section{OPTICAL PROPERTIES UNDER LOW-INTENSITY CW EXCITATION}

\section{A. Sample characterization}

PL and PLE spectroscopy are convenient methods for sample characterization and permit the determination of the $\mathrm{Al}$ composition relative to crossover. Consequently, the sample can be assigned to the direct-gap or indirect-gap regime. PLE is an absorption related technique. It occurs predominantly at the direct band gap due to the oscillator strength associated with this transition $(\Gamma-\Gamma)$. PL, however, occurs at the lowest energy excitonic states due to rapid carrier relaxation. In the case of an indirect-gap semiconductor these states are related to the indirect band gap and consequently, there is a significant energy separation between the PL and PLE resonances.

Figure 1 shows the PL and PLE spectra of the three samples. While the PL peak positions differ from each other by less than $8 \mathrm{meV}$, it can be seen that the absorption edges of samples 1 and 2 are separated from that of sample 3 by 75 and $60 \mathrm{meV}$, respectively. Absorption occurs mainly at the direct gap which increases with increasing $\mathrm{Al}$ content by an amount $\sim 7 \mathrm{meV}$ per $\% \mathrm{Al}^{3}$ This is in reasonable agreement with the nominal $\mathrm{Al}$ concentrations of the samples. However, 


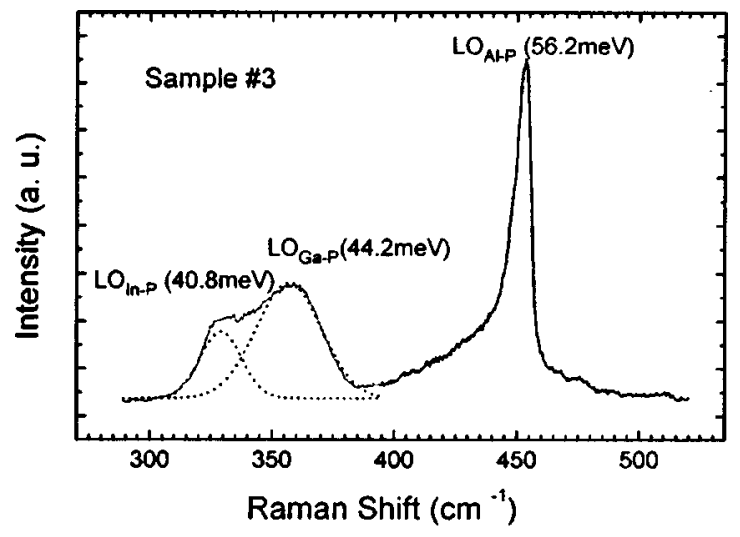

FIG. 2. Raman spectrum of sample 3. Three phonon resonances can be identified.

it should be noted that it is quite difficult to extract the absorption peak positions from the PLE data, since the excitonic resonances in the quaternary system are extremely broad. The absorption resonances may even be broader than indicated by the PLE spectra. As the PLE spectra of all samples show little or no continuum above the excitonic resonances, it can be concluded that nonradiative recombination plays an important role and may produce a narrowing of the excitonic features.

Luminescence occurs at the spectral position of the lowest energy excitonic states. In the present case these are localized indirect excitons (impurity bound or localized at potential fluctuations) as indicated by the phonon replica which will be discussed below. If we consider a localization depth of $\sim 10 \mathrm{meV}^{8}$ and a difference of a few meV between the Rydberg energies at the direct and the indirect gap caused by the different effective masses in each conduction band minimum, ${ }^{9,10}$ we obtain a separation of the band gaps which is considerably smaller than the spectral widths of the resonances in the case of samples 1 and 2. In other words, these samples lie in the immediate vicinity of crossover. However, sample 3 unambiguously has an indirect band gap.

Indirect recombination $(X-\Gamma)$ requires an additional process to provide compensation for the different $k$ vectors of the electron and hole. This can be provided, for example, by the participation of a phonon in the recombination process. As a consequence, phonon replicas occur in indirect transitions. In order to identify the low-energy features of the PL as phonon replicas, the phonon energies of the samples have been determined by Raman spectroscopy. Figure 2 shows the Raman spectrum of sample 3 . Three phonon resonances can be seen which are identified as InP-like (41 $\mathrm{meV})$, GaP-like $(44 \mathrm{meV})$, and AIP-like (56 meV) LO phonons, respectively. ${ }^{11}$ We have tried to fit the PL spectrum of sample 3 modeling the zero-phonon line by a Gaussian curve. Three phonon replica are taken into account by shifting the zero-phonon line by amounts corresponding to the energy positions of the Raman resonances. The widths of the lines are kept equal, while the relative heights are varied. However, we find that these three phonons are not sufficient to completely explain the low-energy feature (see Fig. 3). In addition, a fourth contribution, shown by the filled area in

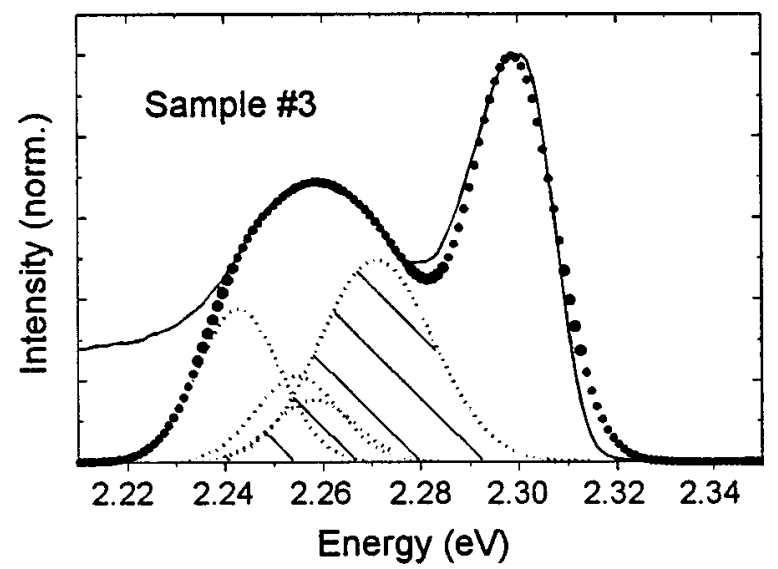

FIG. 3. Measured (solid line) and calculated (circles) PL of sample 3. Three phonon replicas are shown as dotted lines. Impurity-assisted recombination produces a fourth contribution (shown by filled area).

Fig. 3, is required. We consider this fourth contribution to arise mainly from impurity assisted recombination. Since there are contributions to the luminescence at more than 100 meV below the zero-phonon line, we conclude that impurity assisted recombination plays an important role in the present samples. However, it remains unclear whether the participation of acoustic or transverse optical phonons can be completely excluded. The fact that sample 3 shows the strongest phonon replica signal is consistent with its indirect-gap nature.

\section{B. Temperature dependent photoluminescence}

If the energy separation between different conduction band minima is in the range of a few $\mathrm{meV}$ (which corresponds to the thermal energy $k_{B} T$ at moderate temperatures), significant electron populations can occur in the higher valleys. The transfer between these valleys at nonequivalent points of the Brillouin zone occurs via alloy-disorder induced scattering, ${ }^{12}$ i.e., without participation of phonons. For samples near the crossover point, such a multi-valley scenario has a pronounced effect on the temperature dependence of the PL. With increasing temperature, direct recombination gains in relative importance, since the population in the $\Gamma$ valley increases.

In the case of the present crossover samples, direct recombination becomes dominant in comparison to the indirect excitonic PL at $60 \mathrm{~K}$ and produces a strongly broadened peak on the high-energy side of the spectrum (indicated by arrows in Fig. 4). Unlike the conditions at the indirect gap, the Rydberg energy at the direct gap is smaller than the thermal energy at $60 \mathrm{~K}$, i.e., the excitons dissociate and free carrier recombination is observed. The position of these emission bands in samples 1 and 2 is in good agreement with the determination of the direct band-gap energies by PLF.

On the other hand, nonradiative processes produce a strong decrease in the luminescence efficiency when the temperature is raised. This can also be seen in Fig. 4. Due to nonradiative recombination, the PL from localized indirect excitons has decreased by more than one order of magnitude before direct recombination becomes discernible. In the case 


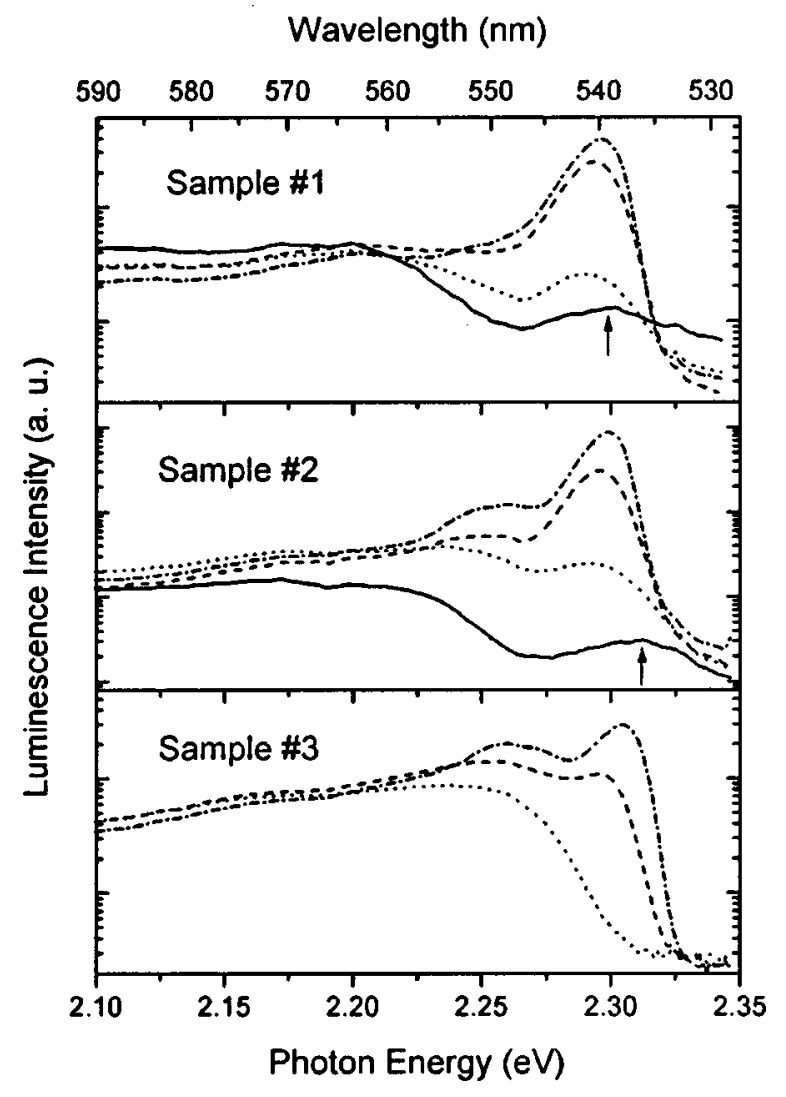

FIG. 4. PL spectra at lattice temperatures of $7 \mathrm{~K}$ (dash-dotted), $20 \mathrm{~K}$ (dashed), $40 \mathrm{~K}$ (dotted), and $60 \mathrm{~K}$ (solid line). Direct band-to-band transitions occur at $T=60 \mathrm{~K}$ (indicated by arrows).

of sample 3, the effect of nonradiative recombination is even stronger. While the excitonic feature has vanished at $40 \mathrm{~K}$ and only impurity assisted luminescence remains, no PL can be observed at $60 \mathrm{~K}$. These results are consistent with an increasing mobility of the indirect excitons and thus an increased probability of reaching nonradiative centers. A similar behavior has already been discussed in connection with the PLE spectra in Sec. III A.

\section{HIGH EXCITATION EFFECTS}

\section{A. Electron-hole plasma in a multi-valley scenario}

Before describing our experimental results, we first explain some important aspects of a dense carrier system in a multi-valley scenario. ${ }^{13}$ At high carrier densities the optical properties of the material are strongly affected by two types of high excitation effects: screening and band filling. ${ }^{14}$ Screening leads to a renormalization of the single-particle energies. In particular, correlation and exchange effects induce spatial rearrangements of the carriers in the dense electron-hole system. These rearrangements result in a significant lowering of the carrier energies. On the other hand, the excitonic binding energy also decreases with increasing carrier density due to screening of the Coulomb interaction. As the reduction of the excitonic binding energy and the renormalization of the band gap are of the same magnitude, the energy position of the excitonic resonance remains constant but only loses oscillator strength. When the energy of

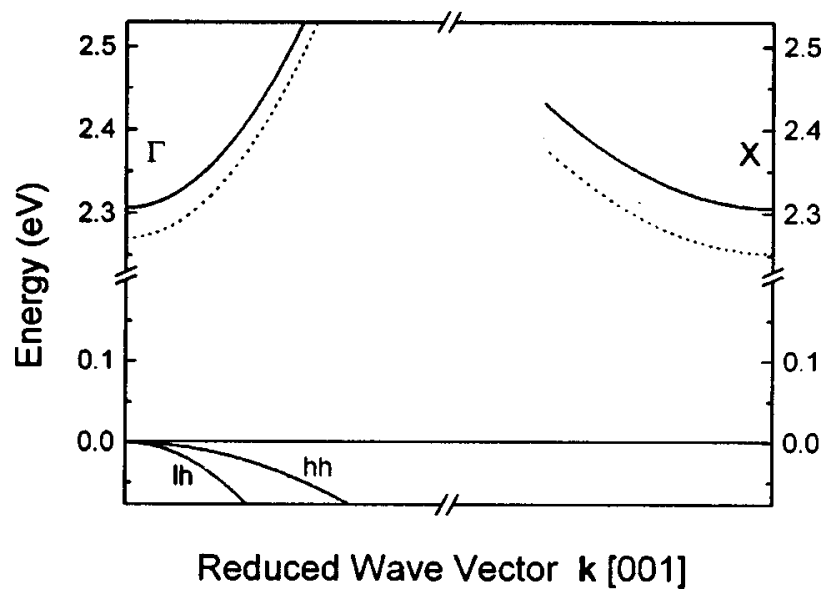

FIG. 5. Typical conduction band alignment of $\left(\mathrm{Al}_{0.5} \mathrm{Ga}_{0.5}\right)_{0.52} \operatorname{In}_{0.48} \mathrm{P}$ in the unexcited case (solid line) and under high excitation (dotted line).

the continuum (or the band-gap energy) falls below the excitonic ground state, the system undergoes a Mott transition, i.e., a transition from an excitonic insulator to a metallic electron-hole plasma (EHP). The corresponding carrier density is known as the Mott density. A further increase of the carrier density leads to an increasing population of the conduction and valence bands with electrons and holes, respectively. This is described by increasing quasi-Fermi levels $f_{e}$ and $f_{h}$, implying that the bands are filling up (band filling). Stimulated emission occurs if population inversion is achieved, i.e., if the condition $f_{e}+f_{h}>1$ is fulfilled.

In the particular case of a multi-valley scenario involving different inequivalent conduction band minima at different points of the Brillouin zone, the distribution of the electrons among these minima is described by a single quasiFermi level and a single temperature. However, at a given quasi-Fermi level, the population and thus the renormalization of a particular valley depend on the corresponding effective electron mass. This means that the band-gap reductions of different inequivalent valleys are different even if the minima are resonant in the unexcited case. An example for such a scenario is illustrated in Fig. 5 which shows the conduction band alignment of $\left(\mathrm{Al}_{0.5} \mathrm{Ga}_{0.5}\right)_{0.52} \mathrm{In}_{0.48} \mathrm{P}$ in the unexcited case and under high excitation. In this particular case the total carrier density is $7.5 \times 10^{18} \mathrm{~cm}^{-3}$. The carrier concentrations in the particular valleys are $n_{\Gamma}=6.3$ $\times 10^{17} \mathrm{~cm}^{-3}$ and $n_{X}=6.9 \times 10^{18} \mathrm{~cm}^{-3}$. The corresponding band-gap reductions are $\Delta E_{x}=54 \mathrm{meV}$ and $\Delta E_{\Gamma}=35 \mathrm{meV}$. It is important to mention that the electron distribution among different minima and the corresponding band-gap reductions must be calculated in a self-consistent manner since the quasi-Fermi level depends on the band-gap energies. ${ }^{15}$

\section{B. Excitation-intensity dependent photoluminescence}

A first approach to studying high excitation effects is provided by excitation-intensity dependent PL experiments. It is important to recall the fact that our measurements have been performed using very short excitation pulses (pulse duration $100 \mathrm{fs})$. The maximum energy density of $345 \mu \mathrm{J} \mathrm{\textrm {cm } ^ { - 2 }}$ corresponds to a carrier density of $\sim 3 \times 10^{18} \mathrm{~cm}^{-3}$ immedi- 

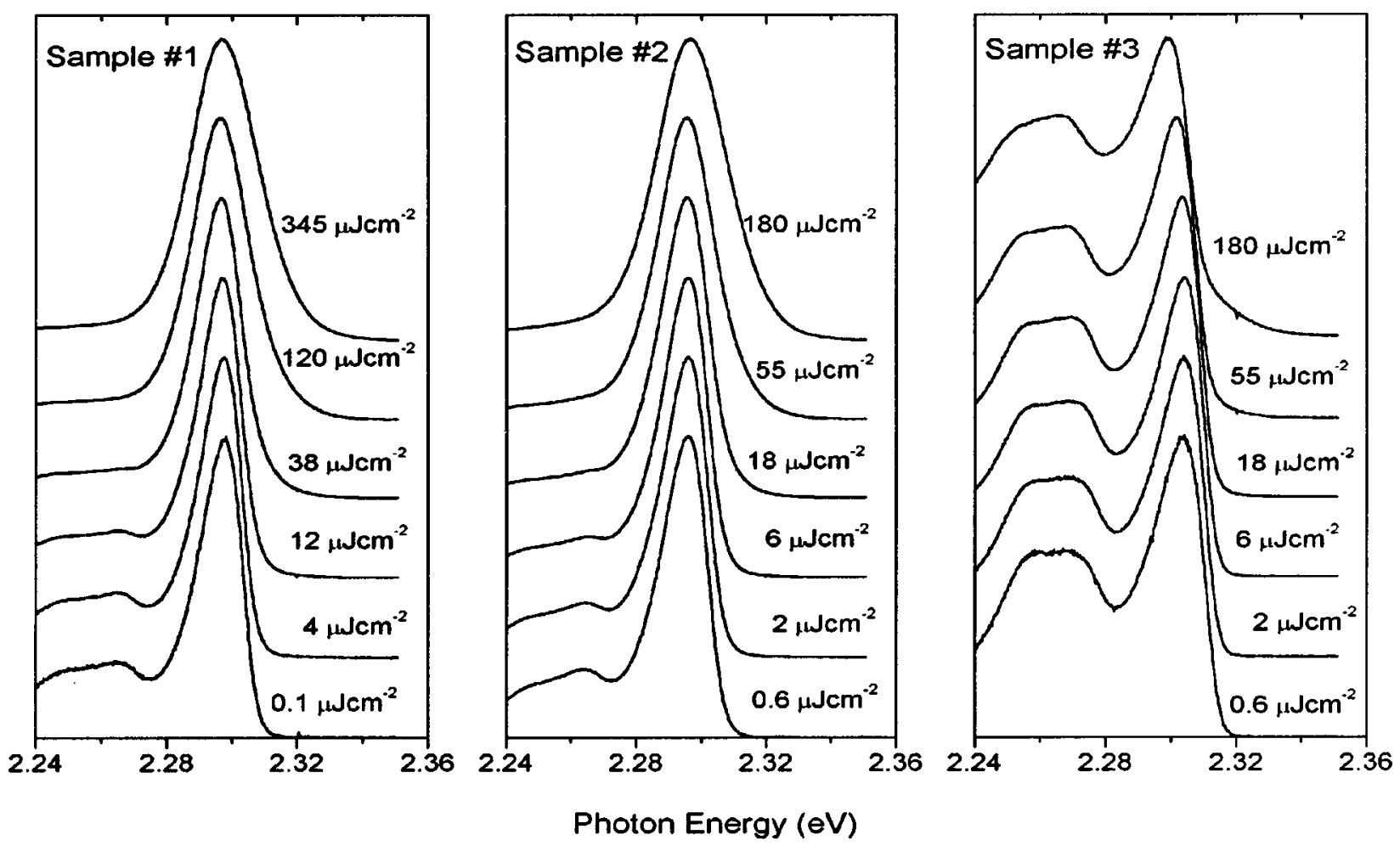

FIG. 6. PL spectra for various excitation energy densities.

ately after excitation. This value is certainly greater than the Mott density which is expected to lie in the range of $\sim 10^{16}-10^{17} \mathrm{~cm}^{-3}$. On the other hand, the carrier densities are expected to be smaller than the Mott density at the lowest energy densities. Thus we should observe a significant change of the luminescence with rising energy density. In practice, the signatures of a Mott transition from localized excitons to an electron-hole plasma can be seen in the case of sample 3 (see Fig. 6). The luminescence spectrum does not change its shape up to an energy density of $10 \mu \mathrm{J} \mathrm{cm}^{-2}$. At higher excitation intensities band-gap renormalization results in a redshift and broadening of the spectra. The phonon replicas shift to the same extent as the zero-phonon line. Due to band-filling effects an additional broadening on the highenergy side occurs at the highest excitation intensity. This broadening is also observed in the case of the crossover samples, but the most striking feature concerning these samples is the fact that the phonon replicas become relatively less intense when the excitation intensity is raised. This indicates that direct recombination $(\Gamma-\Gamma)$ becomes dominant at high excitation intensities. In order to understand this behavior we take into account the fact that the carriers are generated at the $\Gamma$ point. Since both scattering to the $X$ point and thermalization occur on a time scale which is longer than the pulse duration, the $\Gamma$ minimum is strongly populated with hot electrons immediately after excitation. Therefore direct luminescence occurs shortly after excitation. As thermalization becomes slower when the carrier density increases, ${ }^{16}$ direct luminescence gains in importance with respect to the time-integrated spectra when the excitation intensity is raised. Furthermore localization at the $X$ point must be included in our considerations. As discussed in Sec. III B, non- radiative recombination dominates the radiative process when the carrier mobility is increased, due to the thermal energy. A similar situation occurs when the localized states are saturated, i.e., at high carrier densities. While direct luminescence increases with increasing carrier density, an increase of the indirect luminescence is suppressed by nonradiative processes.

\section{Time-resolved photoluminescence}

In order to identify the various recombination processes, we have carried out experiments with picosecond excitation in conjunction with time-resolved detection. Since the repetition rate of the laser system is low, high pulse energies are required in order to obtain a reasonable luminescence signal. In this experiment we used pulses of $20 \mathrm{~mJ} \mathrm{~cm}^{-2}$, i.e., the carrier densities immediately after excitation are very high. Figure 7 shows PL spectra of all samples at different times after picosecond excitation. The maximum of the excitation pulse defines zero on the time axis.

Although sample 3 unambiguously has an indirect band gap, luminescence from the direct gap is observed under these conditions. At $t=0 \mathrm{ps}$, the spectrum is dominated by a contribution with a maximum at $\sim 2.36 \mathrm{eV}$. This contribution stems from direct recombination. The electrons generated at the $\Gamma$ point by direct absorption have the possibility to recombine with holes in the valence band instead of being scattered to the lower energy states at the $X$ point. However, the latter process is very fast and thus this contribution is only observed as long as the excitation pulse is present. This can also be seen very clearly in Fig. 8. The luminescence at $2.358 \mathrm{eV}$ follows the laser pulse exactly, while the lower 

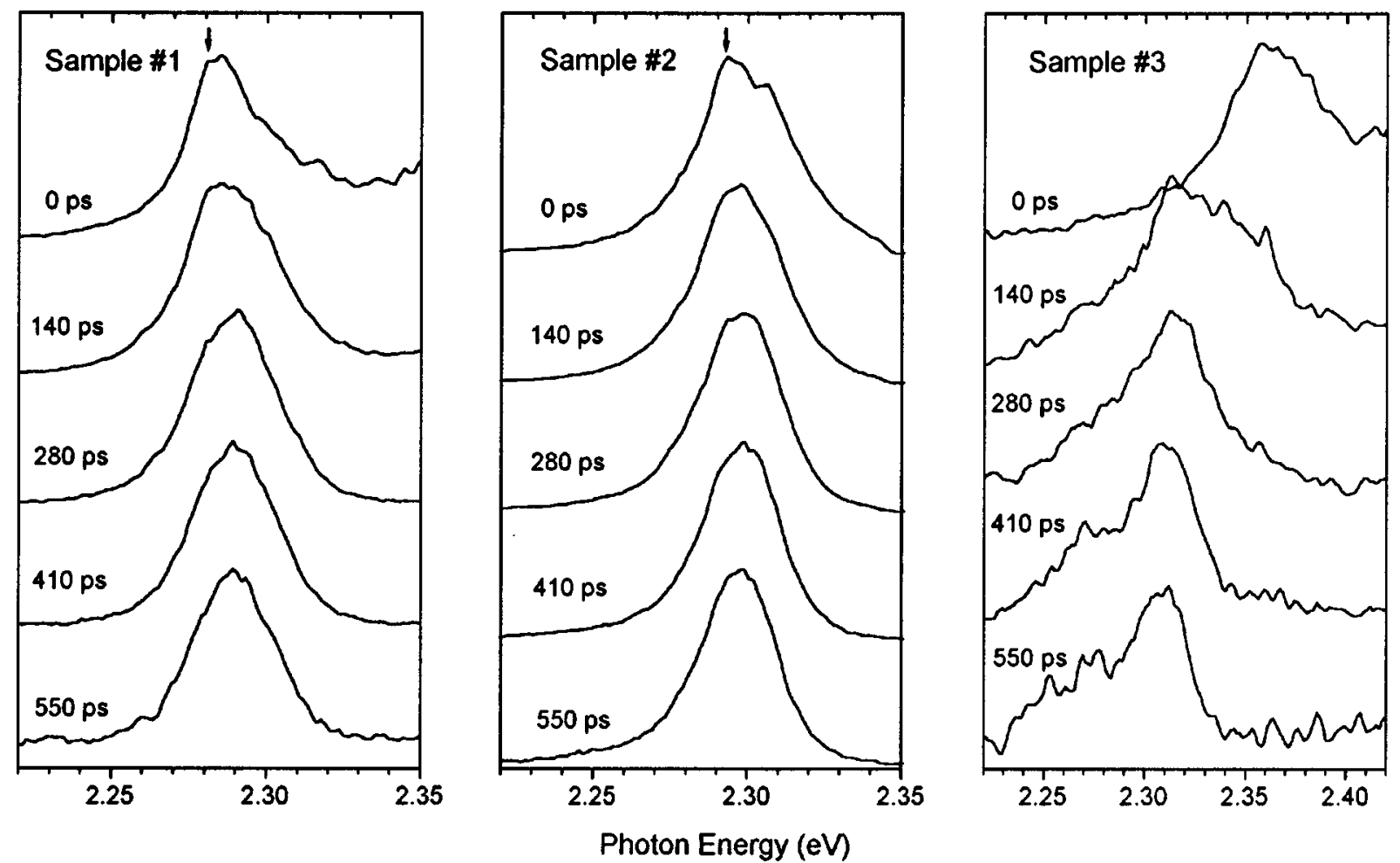

FIG. 7. PL spectra recorded for different times after the excitation maximum. Stimulated emission is labeled by arrows.

energy contribution decays more slowly. The latter is assigned to indirect recombination, phonon replicas on the low-energy side are discernible. As discussed above, indirect recombination is crucial for the luminescence when the carrier density has reached lower values.

In the case of the crossover samples, the energy separation between the different band gaps is relatively smaller than the linewidth of the luminescence. Consequently, one would not expect to observe two contributions that are clearly distinct. Experimentally this is found, but there is an additional interesting feature on the low-energy side, indicated by arrows in Fig. 7. This additional peak decays very rapidly following the laser pulse. As we will see in the following section, it is due to stimulated emission.

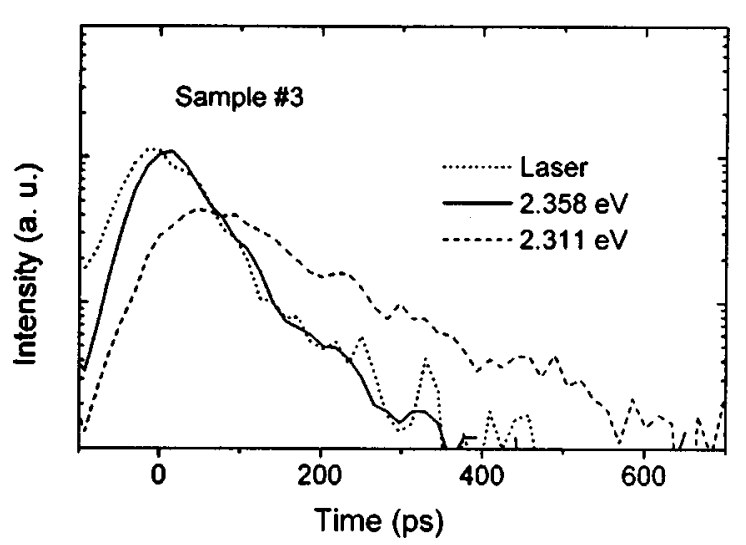

FIG. 8. Temporal evolution of the luminescence at two different energies for sample 3. The time profile of the laser pulse is shown by dotted line.

\section{Stimulated emission and gain}

Our investigations concerning the optical properties of the material for quasistationary excitation and high intensity include two experimental methods: high excitation PL measurements and the variable stripe length method (VSLM). Both were carried out with ns pulses that are long enough to ensure quasistationary conditions. They differ from each other by their excitation and detection geometries. The high excitation PL measurements were carried out in the usual backscattering geometry. Surface emission is detected and provides information concerning the distribution of the carriers at the different conduction band minima, recombination processes, carrier temperatures etc. The VSLM works on the principle of stripe-type excitation in connection with detection of the edge emission. This is a common method for the determination of optical gain. ${ }^{17}$ However, it is sometimes quite difficult to evaluate quantitatively the gain spectra obtained by this method, because saturation effects occur when the stripe length is too large. ${ }^{18}$ On the other hand, the VSLM has an essential advantage: the stripe-type excitation provides a large distance over which light amplification can occur. Stimulated emission increases exponentially with distance along the excitation stripe, while the rise of spontaneous emission is only linear. Consequently, whenever the optical gain exceeds the losses due to reabsorption or scattering the edge emission is characterized mainly by stimulated emission.

For the crossover samples, using the VSLM we find an optical gain of up to $600 \mathrm{~cm}^{-1}$ at excitation intensities of $\sim 350 \mathrm{~kW} / \mathrm{cm}^{2}$. The spectra of the stimulated edge emission (Fig. 9) are very narrow and their spectral positions corre- 


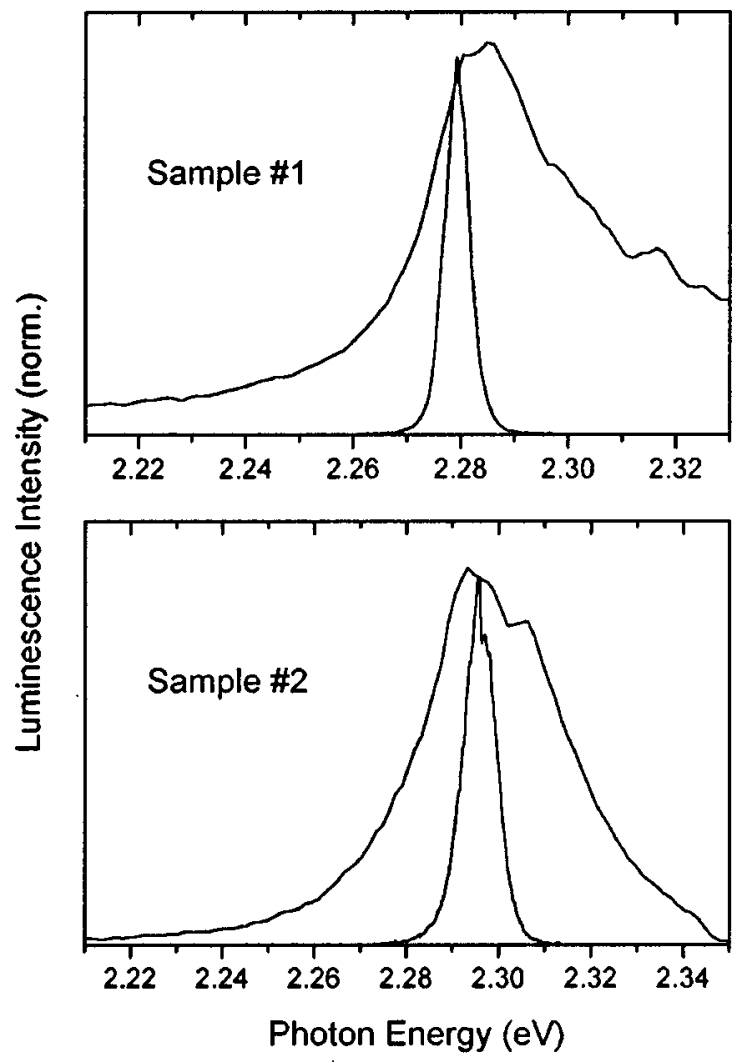

FIG. 9. PL at $t=0 \mathrm{ps}$ (see Fig. 7) and the stimulated edge emission (narrow line) of sample 1 and 2.

spond to the fast decaying peaks in the time-resolved PL spectra that were mentioned in the preceding section. While the threshold for stimulated emission from the crossover samples was determined to be $\sim 300 \mathrm{~kW} / \mathrm{cm}^{2}$, no stimulated emission could be found in the case of sample 3 , even at the highest excitation intensities of several $\mathrm{MW} / \mathrm{cm}^{2}$ that were available in our experimental setup.

In the case of $\mathrm{Al}_{x} \mathrm{Ga}_{1-x} \mathrm{As}$ with $\mathrm{Al}$ contents slightly above crossover, indirect laser emission was found. ${ }^{19-21}$ This very surprising effect could be explained by the fact that alloy disorder provides an efficient mechanism for indirect recombination if the energy separation between direct and indirect band gap is very small. In order to decide whether stimulated emission occurs at the direct or indirect gap in the present material, we recall the fact that direct recombination becomes dominant when the excitation intensity is increased. This is a first indication that the stimulated emission is related to the $\Gamma-\Gamma$ transition.

Figure 10 shows the high excitation PL spectra recorded for excitation intensities of several hundreds of $\mathrm{kW} / \mathrm{cm}^{2}$. Additionally, in each spectrum the stimulated edge emission is also plotted. It can be seen that the stimulated emission of sample 1 lies at a photon energy of $2.28 \mathrm{eV}, 15 \mathrm{meV}$ below that of sample 2 . This is consistent with the energy separation of the unrenormalized direct band gaps which has been discussed above. However, at these excitation intensities band gap renormalization produces strong modifications of the conduction band alignment. This effect must be included in any consideration of the origin of the stimulated emission.

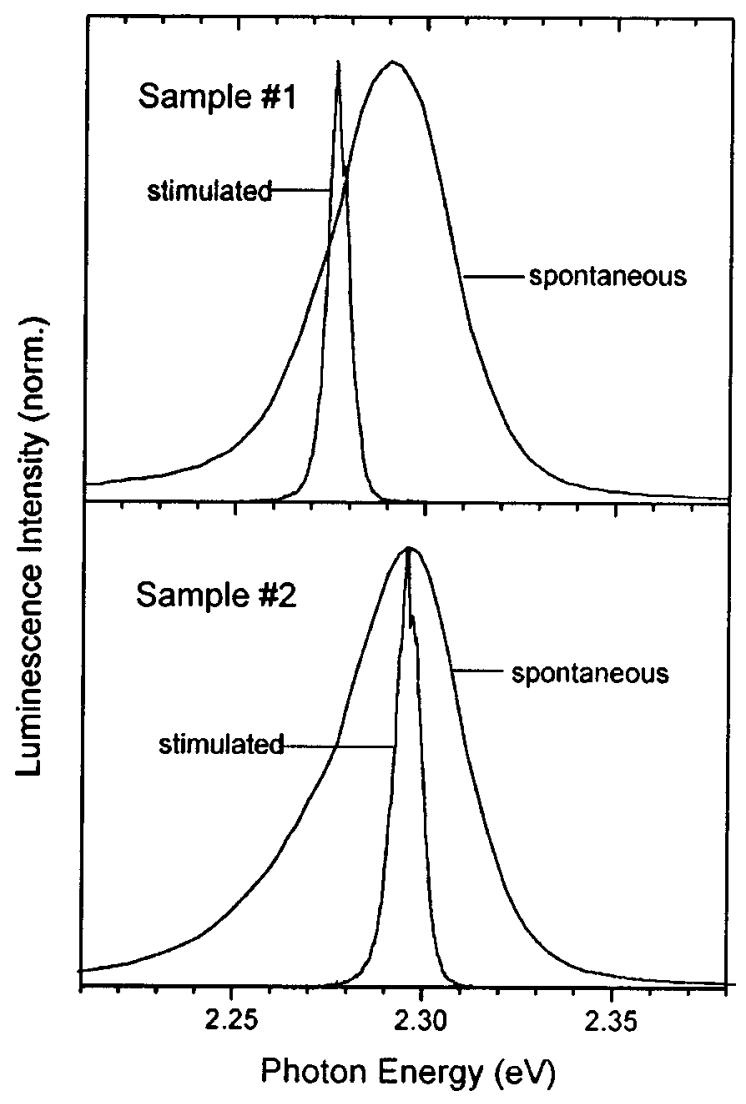

FIG. 10. Spontaneous emission recorded in the backscattering geometry and the stimulated edge emission of samples 1 and 2 .

In order to identify the different contributions to the high excitation PL, we have modeled the line shape by taking into account direct and indirect recombination. Two types of input parameters are required: the effective masses of the $\Gamma$ and the $X$ valleys and the unrenormalized direct and indirect band gaps. The effective masses are taken from literature ${ }^{9,10}$ and the unrenormalized band gaps $E_{g}^{\Gamma}$ and $E_{g}^{X}$ were obtained from our PLE measurements. The fit procedure is as follows: initially arbitrary (but reasonable) values for the total carrier density and the carrier temperature are chosen. With these values the distribution of the carriers within the conduction band minima and the corresponding band gap reductions are calculated in a self-consistent way. Starting from the results of these calculations, the line shape of the luminescence is modeled. The zero-phonon emission of the indirect contribution is calculated using a model without $k$ conservation: three phonon replica, are also considered. For the direct contribution we use a model with $k$ conservation. The ratio between the direct and indirect luminescence is taken as an additional fit parameter (expressed as the scaling factor $F_{\Gamma}$ below) and the spectrum is obtained by adding all contributions. In order to achieve a better agreement with the experimental data, the fit parameters carrier density and temperature are varied and the procedure is repeated in an iterative manner.

There is a good agreement between the calculated and experimental spectra near to the maximum of the emission (see Fig. 11). This part of the spectrum is crucial for the determination of the carrier densities and temperatures. However, in the case of sample 2 the agreement between experi- 

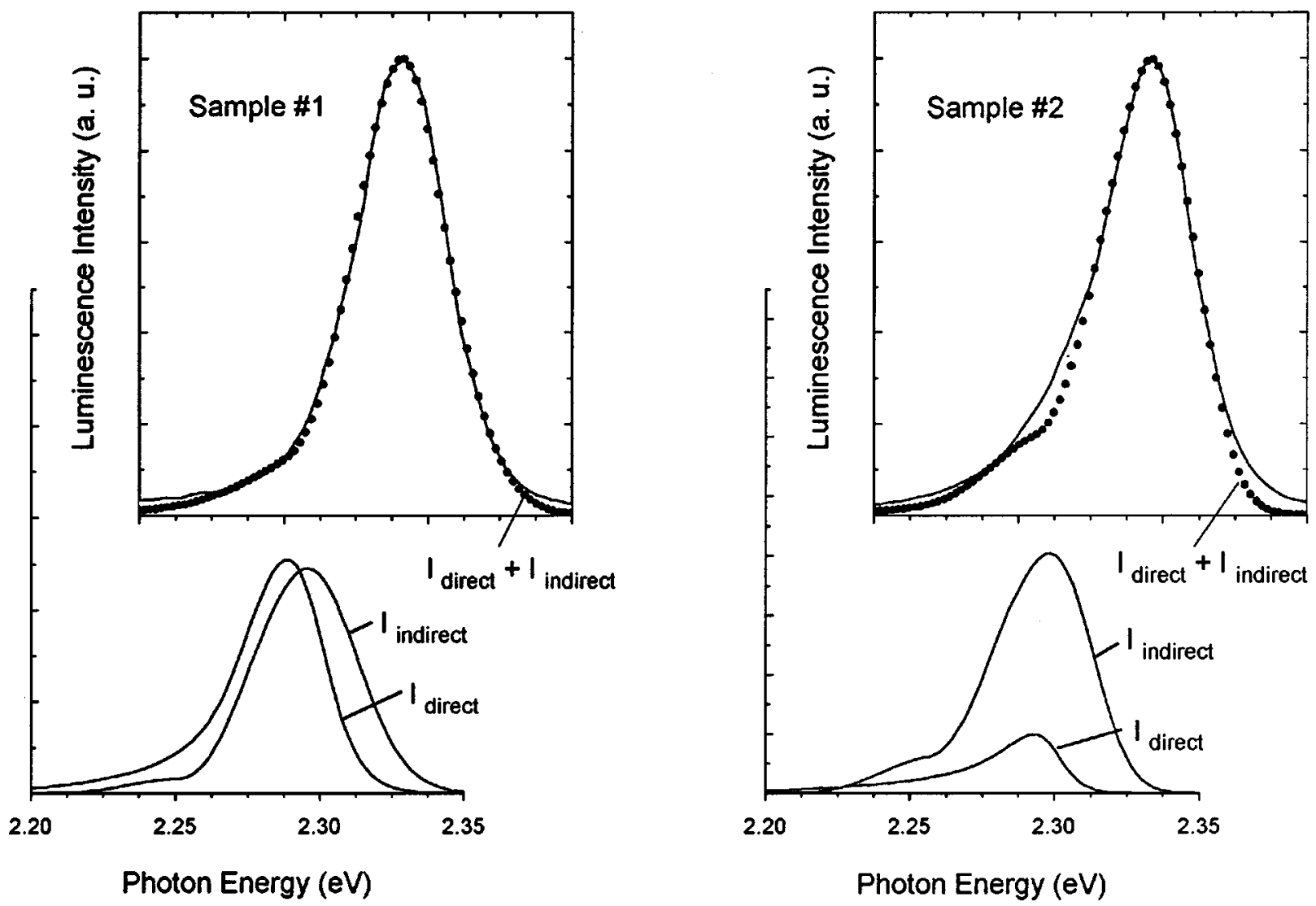

FIG. 11. Upper part: measured (solid line) and calculated (circles) high excitation PL of the crossover samples. Lower part: direct and indirect contributions. The corresponding fit parameters are listed in Table I.

mental and calculated data is not perfect on the low-energy side. As discussed in Sec. III A, impurity-assisted recombination seems to play an important role, but it is not considered explicitly in our model. We have introduced a phenomenological damping in order to avoid an abrupt rise of the direct contribution at the spectral position of the renormalized band gap. The modifications caused by this damping are strongly restricted to the low-energy side, i.e., it has no effect on the results that are obtained from the fit procedure. ${ }^{15}$ For the two crossover samples we obtain carrier temperatures of 60 and $40 \mathrm{~K}$, respectively. These values seem to be reasonable, although the lattice temperature was much lower at 5 $\mathrm{K}$. In fact, one would not expect thermal quasiequilibrium between carriers and lattice since cool carriers recombine and the population of the $X$ valley is maintained by hot electrons from the $\Gamma$ valley. The total carrier densities, $n_{\text {tot }}$, are $7.5 \times 10^{18} \mathrm{~cm}^{-3}$ and $6.75 \times 10^{18} \mathrm{~cm}^{-3}$ for samples 1 and 2 , respectively. As can be seen in Table I, remarkably high carrier concentrations $n_{\Gamma}$ are found in the $\Gamma$ valleys. Since the transition probability for direct recombination is much greater than for indirect recombination, the assumption of direct stimulated emission does not seem to be unreasonable. Furthermore, the ratio between the direct and total luminescence $F_{\Gamma}$ is exactly proportional to $n_{\Gamma} / n_{\text {tot }}$, although this was not preassumed during the fit procedure. The most striking argument for direct stimulated emission is given by its spectral position. As shown in Table I, the renormalized direct band gaps $E_{g \text {,ren }}^{\Gamma}$ correspond very well to the low-energy limit $E_{\text {stim }}^{\min }$ of the stimulated edge emission. On the other hand the indirect gaps of the two samples renormalize to almost the same extent. This is certainly inconsistent with indirect stimulated emission. If indirect recombination was the origin of stimulated emission, one would not expect a remarkable energy separation between the spectra of the two samples. Furthermore, the absolute values of the indirect gaps are 20-30 meV below the low-energy limit of the stimulated emission. In fact it is found that the energy separation between the direct (or indirect) band gaps of the two samples depends very weakly on the total carrier density. From these considerations we conclude that direct recombi-

TABLE I. Fit parameters, unrenormalized and renormalized band gaps and spectral positions of the stimulated edge emission for the crossover samples.

\begin{tabular}{ccc}
\hline \hline & Sample \#1 & Sample \#2 \\
\hline$n_{\text {tot }}\left(\mathrm{cm}^{-3}\right)$ & $7.5 \times 10^{18}$ & $6.75 \times 10^{18}$ \\
$n_{X}\left(\mathrm{~cm}^{-3}\right)$ & $6.87 \times 10^{18}$ & $6.52 \times 10^{18}$ \\
$n_{\Gamma}\left(\mathrm{cm}^{-3}\right)$ & $6.31 \times 10^{17}$ & $2.27 \times 10^{17}$ \\
$T(\mathrm{~K})$ & 60 & 40 \\
$F_{\Gamma}$ & 0.51 & 0.2 \\
$E_{g}^{\Gamma}(\mathrm{eV})$ & 2.306 & 2.318 \\
$E_{g}^{X}(\mathrm{eV})$ & 2.306 & 2.309 \\
$E_{g, \text { ren. }}^{\Gamma}(\mathrm{eV})$ & 2.270 & 2.286 \\
$E_{g, \text { ren. }}^{X}(\mathrm{eV})$ & 2.252 & 2.256 \\
$E_{\text {stim }}^{\text {min }}(\mathrm{eV})$ & 2.271 & 2.286 \\
\hline \hline
\end{tabular}


nation is the origin of stimulated emission. In contrast to $\mathrm{Al}_{x} \mathrm{Ga}_{1-x} \mathrm{As}$ at crossover, there is no indication for indirect stimulated emission.

\section{CONCLUSIONS}

We have investigated three $\left(\mathrm{Al}_{x} \mathrm{Ga}_{1-x}\right)_{0.52} \mathrm{In}_{0.48} \mathrm{P}$ epitaxial layers, two of which have compositions in the immediate vicinity of direct-to-indirect crossover, while the third has an indirect band gap. In order to obtain the direct and indirect band gaps, optical characterization was performed using PL, PLE, and Raman techniques. It is found that the excitonic resonances show strong inhomogeneous broadening which is due to spatial fluctuations of the composition and localization effects. Measurements of material exhibiting reduced spectral broadening may clarify further the points discussed in this article. The optical properties of the crossover samples cannot be explained if one takes into account only one type of recombination, i.e., direct or indirect processes. The optical properties are influenced by both types of recombination, and the excitation conditions determine which of them is dominant. At high excitation levels the crossover samples exhibit stimulated emission. This has been verified by two kinds of experimental procedures: (a) determination of the optical gain using the variable stripe length method and (b) time-resolved PL measurements. Stimulated emission is assigned to the direct recombination. In contrast, all experimental results obtained from the indirect-gap sample can be explained by assuming the typical conduction band alignment of an indirect-gap semiconductor. This sample shows no stimulated emission.

\section{ACKNOWLEDGMENTS}

The authors thank J. Geurts of the University of Aachen and J. Hvam of the Mikroelektronik Centret, Technical Uni- versity of Denmark, for their helpful cooperation. The UK work is supported by the Engineering and Physical Sciences Research Council (EPSRC) Grant No. GR K79581.

${ }^{1}$ A. Lott and R. P. Schneider, Jr., Electron. Lett. 29, 1915 (1993).

${ }^{2}$ H. Sugawara, K. Itaya, H. Nozaki, and G. Hatakoshi, Appl. Phys. Lett. 61, 1775 (1992).

${ }^{3}$ D. J. Mowbray, O. P. Kowalski, M. Hopkinson, M. S. Skolnick, and J. P. R. David, Appl. Phys. Lett. 65, 213 (1994).

${ }^{4}$ J. S. Nelson, E. D. Jones, S. M. Myers, D. M. Follstaedt, H. P. Hjalmarson, J. E. Schirber, R. P. Schneider, J. E. Fouquet, V. M. Robbins, and K. W. Carey, Phys. Rev. B 53, 15893 (1996).

${ }^{5}$ A. Gomyo, T. Suzuki, K. Kobayashi, and I. Hino, Appl. Phys. Lett. 50, 673 (1987).

${ }^{6}$ S.-H. Wei and A. Zunger, Appl. Phys. Lett. 90, 662 (1990).

${ }^{7}$ K. Yamashita, T. Kita, H. Nakayama, and T. Nishino, Phys. Rev. B 53, 15713 (1996).

${ }^{8}$ S. P. Najda, A. H. Kean, M. D. Dawson, and G. Duggan, J. Appl. Phys. 77, 3412 (1995).

${ }^{9}$ K. F. Brennan, J. Appl. Phys. 71, 1055 (1991).

${ }^{10}$ C. T. H. F. Liedenbaum, A. Valster, A. L. G. J. Severens, and G. W. T'Hooft, Appl. Phys. Lett. 57, 2698 (1990).

${ }^{11}$ M. Kubo, M. Mannoh, and T. Narusawa, J. Appl. Phys. 66, 3767 (1989).

${ }^{12}$ H. Kalt, W. W. Rühle, K. Reimann, M. Rinker, and E. Bauser, Phys. Rev. B 43, 12364 (1991).

${ }^{13}$ H. Kalt, Optical Properties of III-V Semiconductors, Springer Series Solid-State Science, Vol. 120 (Springer, Berlin, Heidelberg, 1996).

${ }^{14}$ H. Haug and S. Schmitt-Rink, Prog. Quantum Electron. 9, (1984).

${ }^{15}$ H. Kalt and M. Rinker, Phys. Rev. B 45, 1139 (1992).

${ }^{16}$ J. Shah, Ultrafast Spectroscopy of Semiconductors and Semiconductor Nanostructures, Springer Series Solid-State Science, Vol. 115 (Springer, Berlin, Heidelberg, 1996).

${ }^{17}$ K. L. Shaklee, R. E. Nahory, and R. F. Leheny, J. Lumin. 7, 284 (1973).

${ }^{18}$ H. Kalt, M. Umlauff, M. Kraushaar, M. Scholl, J. Söllner, and M. Heuken, Proceedings of the VIII International Conference on II-VI Compounds, Grenoble 1997 (to be published).

${ }^{19}$ H. Kalt, A. L. Smirl, and T. F. Boggess, J. Appl. Phys. 65, 294 (1989).

${ }^{20}$ M. Rinker, H. Kalt, and K. Köhler, Appl. Phys. Lett. 57, 584 (1990).

${ }^{21}$ A. Wörner, R. Westphäling, H. Kalt, and K. Köhler, Appl. Phys. A: Solids Surf. 64, (1997). 